\title{
Model-Based and Model-Free Decisions in Alcohol Dependence
}

\author{
Miriam Sebold $^{a}$ Lorenz Deserno $^{\mathrm{a}, \mathrm{c}}$ Stefan Nebe ${ }^{\mathrm{d}}$ Daniel J. Schad ${ }^{\mathrm{a}}$ \\ Maria Garbusow $^{a}$ Claudia Hägele $^{a}$ Jürgen Keller ${ }^{a}$ Elisabeth Jünger ${ }^{\mathrm{e}}$ \\ Norbert Kathmann $^{b}$ Michael Smolka ${ }^{d}$ Michael A. Rapp ${ }^{f}$ \\ Florian Schlagenhauf ${ }^{a, c}$ Andreas Heinz ${ }^{a}$ Quentin J.M. Huys ${ }^{g, h}$
}

\begin{abstract}
${ }^{a}$ Department of Psychiatry and Psychotherapy, Campus Charité Mitte, Charité Universitätsmedizin Berlin, and
${ }^{b}$ Institute for Psychology, Humboldt-Universität zu Berlin, Berlin, 'Max Planck Institute for Human Cognitive and Brain Sciences, Leipzig, dDepartment of Psychiatry and Psychotherapy, Section of Systems Neuroscience, Technische Universität Dresden, and ${ }^{~}$ Department of Psychiatry and Psychotherapy, University Hospital Carl Gustav Carus at Technische Universität Dresden, Dresden, and ${ }^{\mathrm{f} E x c e l l e n c e ~ A r e a ~ C o g n i t i v e ~ S c i e n c e s, ~ S o c i a l ~ a n d ~}$ Preventive Medicine, University of Potsdam, Potsdam, Germany; ${ }^{9}$ Translational Neuromodeling Unit, Department of Biomedical Engineering, University of Zurich and ETH Zurich, and hepartment of Psychiatry, Psychotherapy and Psychosomatics, Hospital of Psychiatry, University of Zurich, Zurich, Switzerland
\end{abstract}

\section{Key Words}

Alcohol dependence · Decision-making $\cdot$ Reinforcement learning $\cdot$ Dopamine $\cdot$ Computational psychiatry

\begin{abstract}
Background: Human and animal work suggests a shift from goal-directed to habitual decision-making in addiction. However, the evidence for this in human alcohol dependence is as yet inconclusive. Methods: Twenty-six healthy controls and 26 recently detoxified alcohol-dependent patients underwent behavioral testing with a 2-step task designed to disentangle goal-directed and habitual response patterns. Results: Alcohol-dependent patients showed less evidence of goal-directed choices than healthy controls, particularly after losses. There was no difference in the strength of the habitual component. The group differences did not survive controlling for performance on the Digit Symbol Substitution Task. Conclusion: Chronic alcohol use appears
\end{abstract}

to selectively impair goal-directed function, rather than promoting habitual responding. It appears to do so particularly after nonrewards, and this may be mediated by the effects of alcohol on more general cognitive functions subserved by the prefrontal cortex.

(c) 2014 S. Karger AG, Basel

\section{Introduction}

Substance dependence is characterized by maladaptive choices that contrast with the subjects' explicitly stated desires, such as the failure to abstain despite the desire to quit drinking. This inflexible and compulsive behavior in addiction suggests a failure or disruption of several components of the underlying decision-making systems.

Two well-defined components of decision-making have been computationally characterized. On the one hand, a flexible, goal-directed, model-based planning sys-

\section{KARGER}

E-Mail karger@karger.com

www.karger.com/nps
C 2014 S. Karger AG, Basel

0302-282X/14/0702-0122\$39.50/0 
tem explicitly considers the consequences of actions [1]. It is 'model-based' in that it relies on a model of the world (in simple experiments often just the action-outcome contingencies), and then deduces from this model the best sequence of actions. Although it is computationally costly, requiring the explicit consideration of future outcomes, it can be immediately sensitive to environmental changes and lead to rapid behavioral adaptation. In parallel, a more rigid habitual system repeats actions that were in the past associated with reward $[2,3]$. The learning process that leads to habits relies on iterative updates of expectations through putatively dopaminergic [4-6] prediction errors, but unlike the goal-directed system does not rely on an explicit model. Hence, it is termed modelfree. Because it relies on iterative updating, it is also slow, requiring substantial and repeated experience before behavioral adjustments. Tasks that require rapid shifting between behavioral strategies have been used to distinguish model-based and model-free components behaviorally and neurobiologically in humans and animals. Examples of such tasks are devaluation or motivational shift experiments, whereby the signature of habitual responding is a continued responding for an outcome that is no longer desired. This is reminiscent of drug taking persisting in the face of negative consequences.

Numerous studies in animals have shown that drugs of abuse shift the balance towards habits. Studies using for instance devaluation paradigms [4-7] have shown persistence of responding increasing with alcohol. The processes that speed up habituation have been suggested to also facilitate transformation into compulsions [8-13]. Work in humans with addictions has also shown evidence of inflexible choices, even in terms of non-drug-related rewards such as monetary gains. For instance, alcohol- [14] and stimulant-dependent [15-17] patients show impairments in shifting their responses in probabilistic reversal learning and fail to adapt their responses after errors in stop signal tasks $[18,19]$. Moreover shifts towards automatic action tendencies temporally precede relapses [20] and can be trained to improve treatment outcome [21]. There have also been attempts to directly examine how substance dependence affects the relationship between goal-directed and habitual control in humans. A satiety devaluation paradigm did not reveal evidence of habitual responding for either cigarettes or chocolate in smokers, suggesting that not all responses for drug-related stimuli are necessarily habitual in drug users [22]. Interestingly, however, alcohol expectancy [23] and acute alcohol administration [24] did reduce the effect of satiety devaluation, suggesting that alcohol can specifically impair goal-

Decision-Making after Chronic Alcohol Intake directed decisions. Indeed, the goal-directed system is likely to depend on the kind of cognitive processes that are known to be impaired by alcohol $[25,26]$. Sjoerds et al. [27] used an instructed devaluation in a slip of action tasks which involves complex relationships between stimuli, outcomes and responses. They found evidence for a shift towards habits in alcohol-dependent patients, which was accompanied by an increased functional magnetic resonance imaging signal in habit-related areas like the posterior putamen [28] and a decreased signal in goaldirected ventromedial prefrontal and anterior putamen areas [29-31], which parallels findings in animals [32].

Devaluation experiments involve single, sudden and large changes. On the one hand, these changes are obvious to human subjects and such salient changes might therefore lead to potent re-engagement of flexible goaldirected behavior. On the other hand, the slip of action task is very complex, and the performance of goal-directed decisions might be hampered by impairments affecting upstream cognitive functions including working memory [33]. We here use a third type of task [31] that has been developed based on computational arguments about the statistical efficiency of habitual and goal-directed systems [3]. It examines the relative contributions of habitual and goal-directed choices using continuous, subtle valuation shifts rather than few large or instructed ones. This task is also particular in that it allows us to compare the consequences of gains and nongains. Furthermore, given the sensitivity of goal-directed choices to cognitive load [25] and the recently reported importance of other cognitive measures [33,34], we also examine whether any effects of alcohol dependence on the structure of decision-making might be accounted for by differences in more general cognitive measures.

\section{Methods}

Twenty-six recently detoxified alcohol-dependent patients (5 females) and 26 healthy comparison subjects ( 5 females) participated in this study. Demographic and clinical group characteristics of the final sample are outlined in table 1. Groups were matched for age, gender and years of education. All participants were examined for past and present psychiatric disorders using the Screening Version of the Structured Clinical Interview for DSM-IV [35]. All patients fulfilled DSM-IV criteria for alcohol dependence without axis I comorbidity. The days of alcohol abstinence before study participation in the patient group ranged from 2 to 39 with a mean of $15.1 \pm 10.4$ days. All healthy controls had no current or past major psychiatric disorder. All participants had normal or corrected-to-normal vision, and were also screened for neurological diseases. After detailed verbal and written instruction on the procedures of the study, participants gave their 
Table 1. Demographic and clinical characteristics of 26 patients and 26 matched controls

\begin{tabular}{|c|c|c|c|c|c|c|}
\hline & \multicolumn{2}{|c|}{ Number } & \multicolumn{2}{|l|}{ Mean } & \multirow[t]{2}{*}{$\mathrm{t}$ values } & \multirow[t]{2}{*}{$\mathrm{p}$} \\
\hline & ALC & $\mathrm{HC}$ & ALC & $\mathrm{HC}$ & & \\
\hline Age, years & 26 & 26 & 44.0 & 43.3 & $\mathrm{t}_{50}=0.304$ & 0.76 \\
\hline Education, years & 26 & 25 & 11.0 & 11.5 & $\mathrm{t}_{49}=1.39$ & 0.17 \\
\hline Verbal IQ (WST) & 25 & 26 & 100.5 & 106.0 & $\mathrm{t}_{49}=1.92$ & 0.061 \\
\hline Cognitive speed (DSST) & 25 & 24 & 59.0 & 69.9 & $\mathrm{t}_{47}=2.18$ & 0.035 \\
\hline Verbal working memory (DS) & 25 & 25 & 6.8 & 7.5 & $\mathrm{t}_{48}=1.06$ & 0.29 \\
\hline Executive functioning (TMT-A) & 19 & 24 & 33.5 & 32.4 & $\mathrm{t}_{41}=0.32$ & 0.75 \\
\hline Executive functioning (TMT-B) & 19 & 24 & 74.5 & 72.3 & $\mathrm{t}_{41}=0.18$ & 0.86 \\
\hline Verbal memory (word list) & 19 & 24 & 8.2 & 8.9 & $\mathrm{t}_{41}=1.14$ & 0.26 \\
\hline
\end{tabular}

Each group included 5 females. Comparisons are based on independent-sample t tests. $\mathrm{p}$ values indicating statistical significance at a level less than 0.05 are displayed in italics.

ALC = Alcohol-dependent patients; HC = healthy controls; verbal IQ assessed by German vocabulary test (Wortschatztest [39]); cognitive speed assessed by Digit Symbol Substitution Test (DSST) from Wechsler WAISR [40]; verbal working memory assessed by digit span (DS) backwards test [40]; executive functioning assessed by trail making test (TMT) A and B [41]; verbal memory assessed by word list from the Consortium to Establish a Registry for Alzheimer's Disease [42].

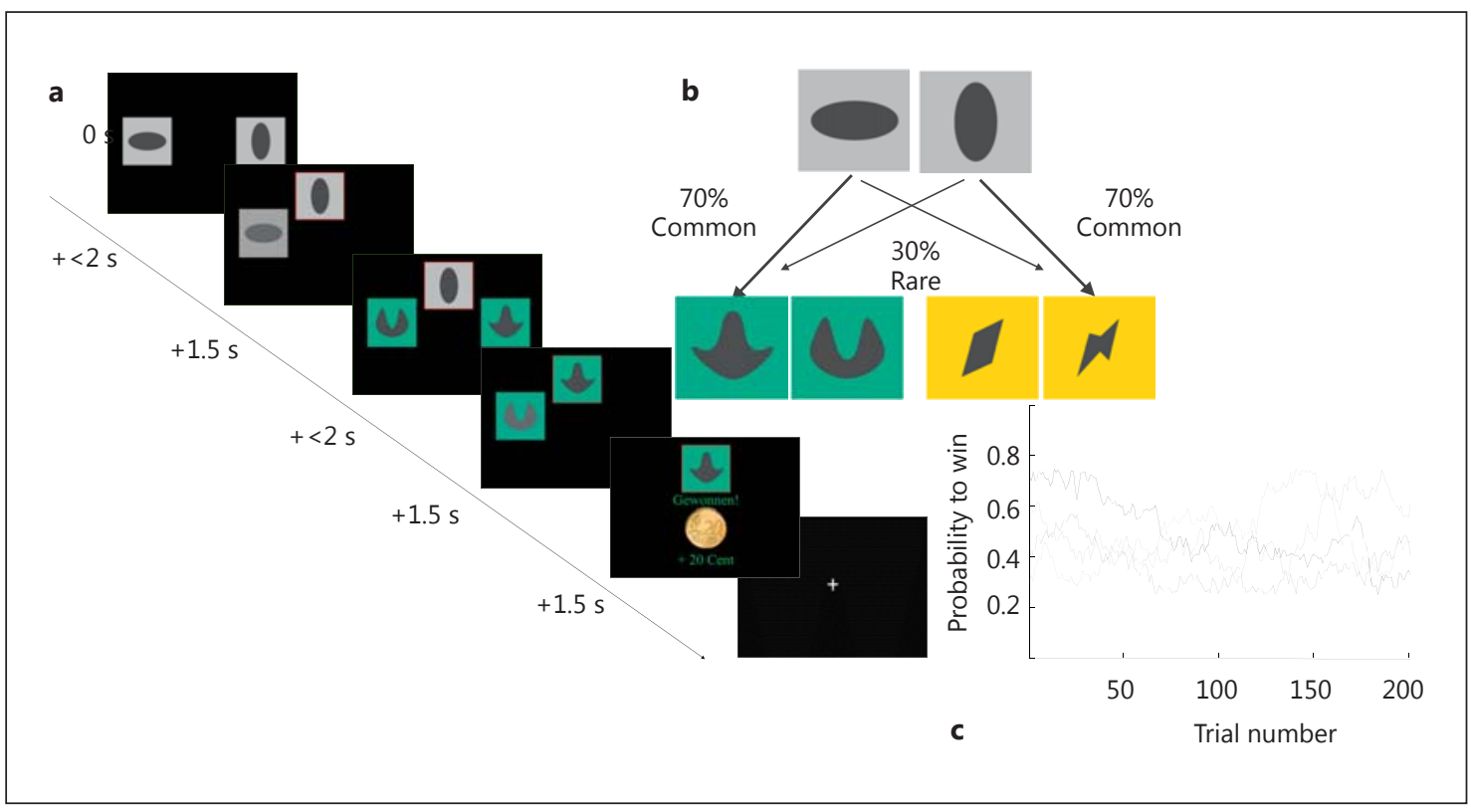

Fig. 1. a Trial configuration for the experiment. Each trial consisted of 2 different stages and each stage involved a choice between 2 stimuli. In the first stage, subjects chose between 2 abstract stimuli on a gray background. The chosen stimulus was highlighted by a red frame and moved to the top of the screen, where it remained visible for $1.5 \mathrm{~s}$; at the same time, the other stimulus faded away. Subjects then reached a subsequent second stage. Here subjects saw 1 of 2 further pairs of colored stimuli and again chose between these. The monetary outcome following this second-stage choice (gain or no gain of 20 cent) was then presented centrally on the screen. $\mathbf{b}$ One pair of colored second-stage stimuli occurred com- monly (on 70\% of trials; 'common trials') after choice of one firststage gray stimulus, while the other second-stage pair was equally strongly associated with the other first-stage stimulus (common). On the remaining $30 \%$ of trials, the chosen first-stage option resulted in a transition to the other second-stage stimulus pair (rare). c Reinforcement probabilities for each second-stage stimulus changed slowly and independently according to gaussian random walks with reflecting boundaries at 0.25 and 0.75 . Win probabilities are displayed as a function of trial number, according to Daw et al. [31]. 
Fig. 2. First-stage behavior as a function of reward and frequency of the previous trial for pure model-free versus pure modelbased strategies. A pure model-free decision-making strategy would lead to a main effect of reward (a), but no effect of or interaction with frequency, while a pure model-based decision strategy would lead to an interaction between reward and frequency without a main effect of reward (b).

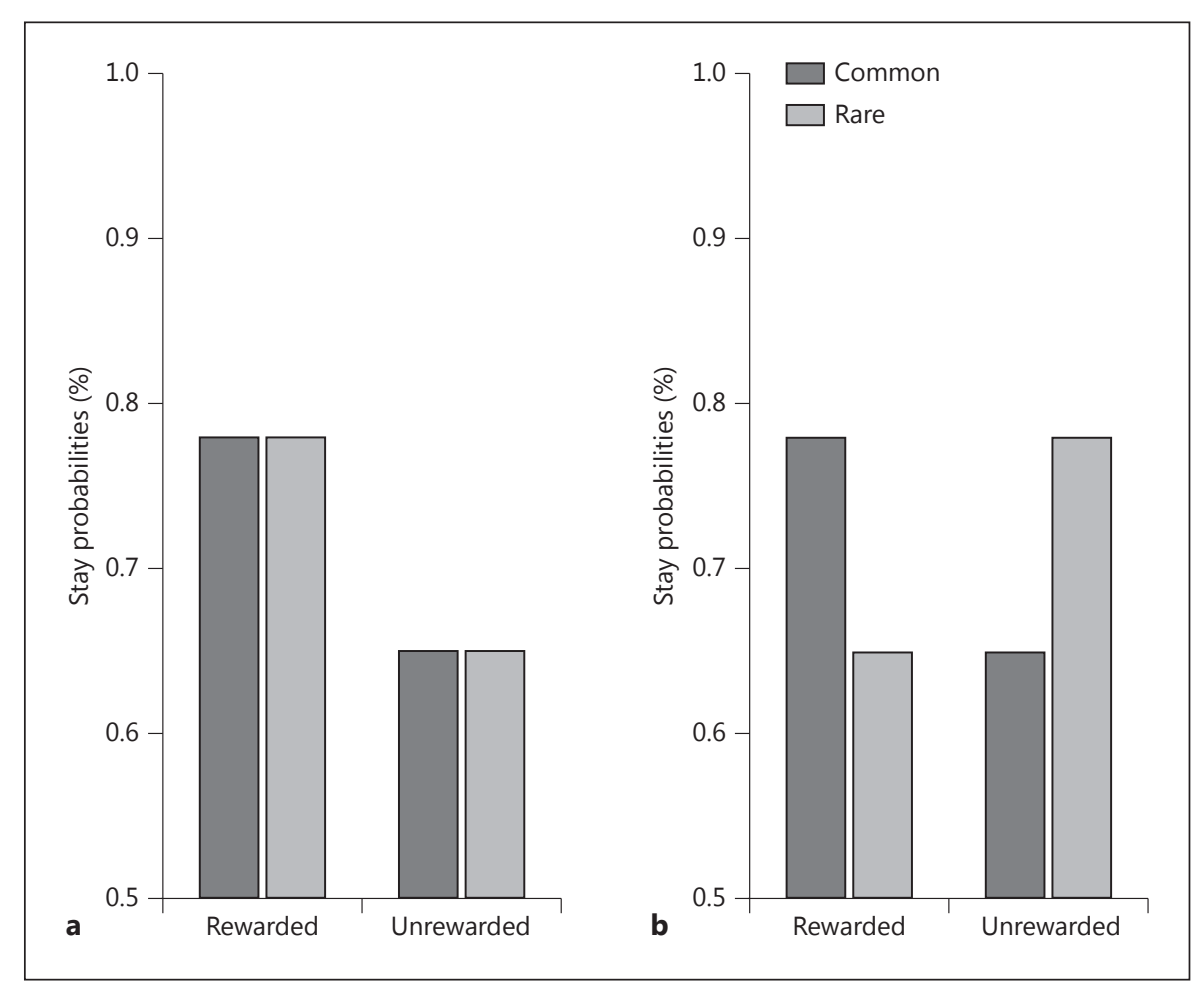

written informed consent. The study was approved by the local ethics committee of Charité Universitätmedizin Berlin and Universitätklinikum Dresden.

\section{Task and Procedure}

All participants underwent neuropsychological testing including standard tests assessing crystallized intelligence, cognitive speed, memory and executive functioning (table 1). Participants additionally performed the 2-stage Markov decision task as described by Daw et al. [31] in 2011 (see fig. 1 for a detailed task description).

The task was reprogrammed in MATLAB, using the Psychophysics Toolbox extensions $[36,37]$ and used different stimuli. Prior to the experiment, participants were explicitly informed about the task structure. Critically, the very detailed subject instructions were carefully translated from the English version. Participants were told that the transition matrix from first-step choices to second stages would stay constant and that the selection of one stimulus on the first stage would lead to a particular stimulus pair on the second stage more often than it would lead to the other second-stage stimulus pair. Participants were instructed that second-stage reward probabilities were independent of each other and would slowly change over the course of the experiment. Participants were familiarized with the paradigm before the task by performing a shortened version of the paradigm (50 trials) with different reinforcement probabilities and a different stimulus set. They were instructed to maximize their monetary outcome throughout the experiment. Participants' overall payout was EUR 13 plus the accumulated reward of one third of all trials. The maximal payout was limited to EUR 20. The task consisted of 201 tri- als. Trials were separated by an exponentially distributed intertrial interval, ranging between 1 and $7 \mathrm{~s}$. The maximum response time was $2 \mathrm{~s}$ for first- and for second-stage choices. If participants failed to make a response in this time window, the German phrase for 'too slow!' appeared on the screen for $2 \mathrm{~s}$, and the trial was aborted. The two stimuli at the first and second stage were assigned randomly between left and right from trial to trial.

\section{Behavioral Analysis}

We performed a simplified analysis focusing only on first-stage choices. Model-based and model-free strategies predict different patterns of first-stage choices. A model-free strategy predicts purely reinforcement-guided action selection: first-stage choices should be repeated after a previous trial's second-stage choice had resulted in reward whereas a first-stage switch should occur after a previous trial had ended up being not rewarded. Thus, modelfree action selection should occur irrespectively of whether the transition to the second stage in the previous trial was a common or a rare one (fig. 2a). In contrast, model-based action selection includes the consideration of the task structure in its transition. This results in an inverted response behavior following rare trials. Consider a trial in which a first-stage response uncharacteristically results in a second-stage stimulus pair to which it usually does not lead (rare) and in which the second-stage selection is then rewarded. Model-based action selection would then predict a decreased probability of choosing this first-stage stimulus again, as the selection of the first-stage stimulus that has initially not been chosen has a higher likelihood of leading to the rewarded secondstage stimulus pair (common; fig. 2b). 


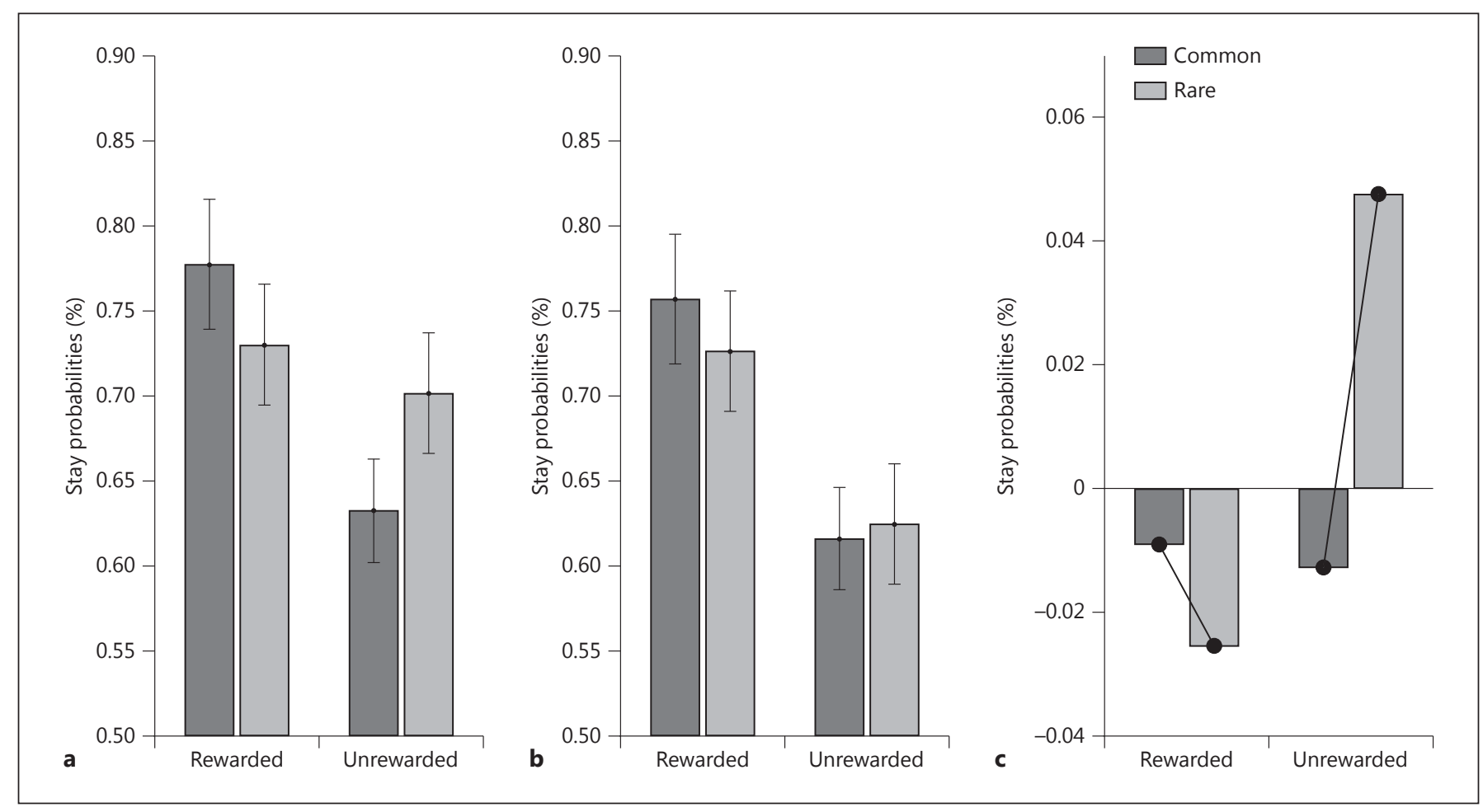

Fig. 3. Observed first-stage behavior as a function of reward and frequency of the previous trial for healthy controls (a) and alcohol-dependent patients (b). Error bars represent standard errors of means. c Standardized differences in stay probabilities between healthy controls and alcohol-dependent patients.

The major aim of this investigation was to specifically assess whether alcohol-dependent patients displayed a shift from model-based to model-free control. For this purpose we calculated 2 individual scores, one for model-based and one for model-free control. Individual model-free scores reflected the individual main effect of reward (\% reward common $+\%$ rewarded rare $\%$ unrewarded common - \% unrewarded rare), whereas individual scores for model-based control reflected the interaction between transition frequency and reward (\% reward common + $\%$ unrewarded rare - \% rewarded rare - \% unrewarded common). We computed a 1-tailed t test in each group (which compares both scores in each group against zero), in order to test whether both decision-making systems were evident within each group.

We then conducted independent-samples t tests to assess our a priori hypotheses that model-free contributions would be larger in alcohol-dependent patients than in healthy controls and conversely that model-based contributions would be larger in healthy controls than in alcohol-dependent patients. As we had explicit hypotheses on the direction of within-group and between-group effects of both model-free and model-based control we tested all comparisons regarding these hypotheses against a 1-tailed criterion.

In order to assess whether the neuropsychological variable that differed between groups (Digit Symbol Substitution Test, DSST) had an influence on model-free or model-based control we first regressed the outcome variables (total earnings and individual model-based scores) onto the neuropsychological variable, and performed independent-samples t tests on the residuals. All statistical analyses were performed using MATLAB version R2007a [38].

\section{Results}

\section{Participants}

Participant characteristics are shown in table $1 . \mathrm{Pa}$ tients and controls were matched for age, sex and education. Nevertheless, there was a significant difference in one measure of cognitive functioning (DSST, p < 0.05), and groups showed a tendency towards differing on verbal IQ (German vocabulary test, Wortschatztest: $\mathrm{p}=$ 0.061).

Participants rarely missed any responses (mean = $1.8 \%, \mathrm{SD}=2.43 \%$ ). Missed trials were omitted from analysis. Patients and controls did not differ in their average stay/switch behavior at the first stage (no group difference in overall probability of stay/switch $t_{50}=0.65, p=$ $0.52)$. 


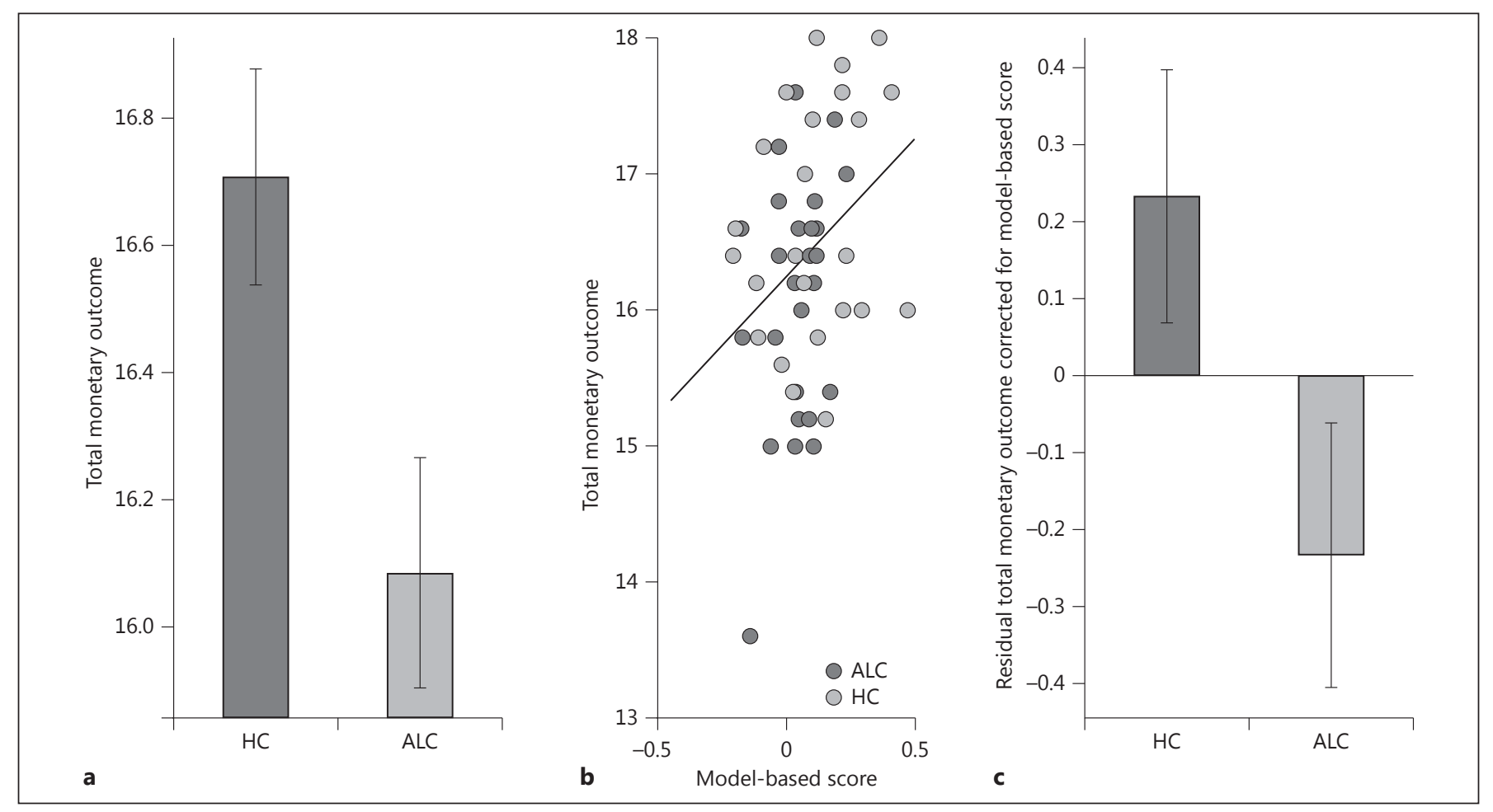

Fig. 4. Monetary outcome. $\mathrm{HC}=$ Healthy controls; $\mathrm{ALC}=$ alcohol-dependent patients. a Group differences in total monetary outcome. b Correlation between total monetary outcome and individual model-based scores. c Total monetary outcome corrected for differences in model-based scores.

\section{Model-Free Choices}

Both groups showed significant model-free influences in their choice behavior (model-free scores greater than zero, alcohol-dependent patient 1 -tailed $\mathrm{t}$ test, $\mathrm{t}_{25}=5.03$, $\mathrm{p}<0.001$; healthy control 1-tailed $\mathrm{t}$ test, $\mathrm{t}_{25}=4.1 \mathrm{p}<$ 0.001 ). Thus, subjects tended to choose the same firststage stimulus when rewarded in the previous trial, but switched to the opposing first-stage stimulus when not rewarded. Against our prediction, patients did not show a stronger model-free component (individual model-free scores alcohol-dependent patients vs. healthy controls 1 -tailed $\mathrm{t}$ test, $\mathrm{t}_{50}=1.08, \mathrm{p}=0.14$ ).

\section{Model-Based Choices}

Goal-directed components were present in both groups (individual model-based score greater than zero; 1-tailed tests: healthy control $\mathrm{t}_{25}=3.32, \mathrm{p}<0.001$, alcohol-dependent patient $\left.\mathrm{t}_{25}=1.95, \mathrm{p}<0.05\right)$. Hence, switches tended to occur when the outcome of the previous trial was a reward, but the transition rare, or when the outcome was a nonreward and the transition a common one. Healthy controls used significantly more model-based strategies than alcohol-dependent patients, as indicated by between-group differences in individual model-based scores $\left(t_{50}=1.90, p<0.05\right.$, 1-tailed).

Figure 3 displays the stay probabilities for healthy controls (fig. 3a), alcohol-dependent patients (fig. 3b) and between-group differences in stay probabilities for all 4 trial types (fig. 3c). Visual inspection suggests that the groups mainly differed in stay probabilities after unrewarded trials. In order to test for differential betweengroup differences in model-based choice behavior for unrewarded and rewarded trials, we calculated individual model-based scores for unrewarded and rewarded trials separately and used independent-sample $t$ tests to compare these between groups. These post hoc t tests confirmed that controls modulated their responses after losses according to a model-based strategy (i.e. were sensitive to transition frequency) more than patients (1-tailed test: $\left.t_{50}=2.27, p<0.05\right)$. However, modelbased control after rewarded trials was not greater in controls than patients (1-tailed test: $\mathrm{t}_{50}=0.57, \mathrm{p}=0.28$ ). Thus, the difference in terms of model-free/model- 
Fig. 5. Group differences in model-based scores (a) and model-based scores corrected for DSST (b). HC = Healthy controls; ALC $=$ alcohol-dependent patients.

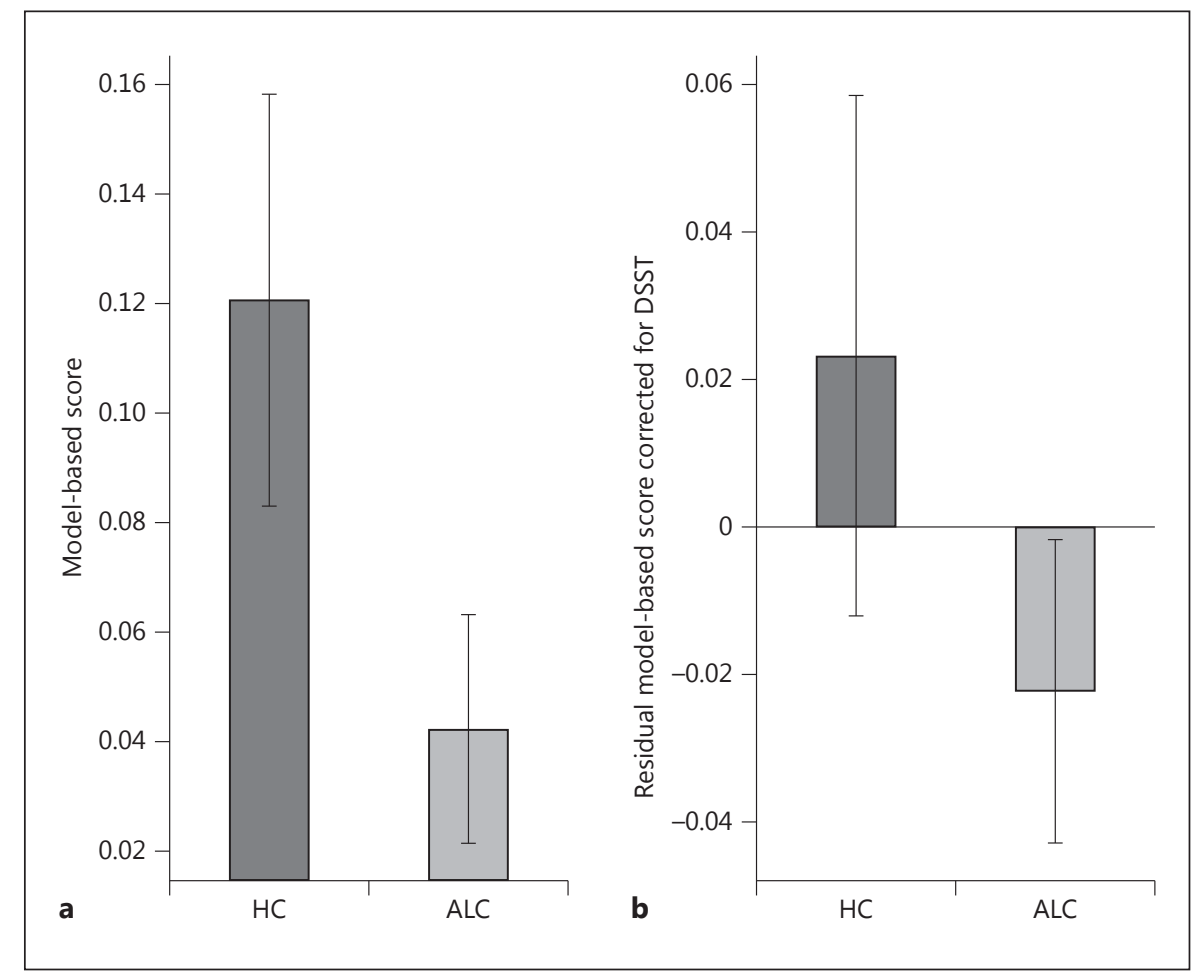

based choices between controls and patients was driven by a failure of goal-directed control after nonrewards in patients.

On average, subjects earned EUR 16.4 (total range 13.60-18.00). Patients earned less money than controls (2-tailed t test, $\mathrm{t}_{50}=2.53, \mathrm{p}<0.05$, fig. $\left.4 \mathrm{a}\right)$. As the optimal response strategy exploits the structure of the task, model-based responding was expected to improve the overall outcome. Individual model-based scores were indeed positively correlated with the total monetary outcome (Pearson's $\mathrm{r}=0.33, \mathrm{p}<0.05$; fig. $4 \mathrm{~b}$ ). As expected, total outcome was not related to the score for model-free behavior (Pearson's $r=-0.13, p=0.36$ ). However, the group differences in earnings remained marginally significant after correcting for model-based scores $\left(\mathrm{t}_{47}=1.96, \mathrm{p}=\right.$ 0.06 ), indicating that goal-directedness only partially accounted for between-group differences in total monetary outcome (fig. 4c).

Effect of Cognitive Measures on Model-Based Behavior Despite carefully matching for education, groups differed on the DSST - a measure of cognitive speed (table 1). We therefore asked whether the apparent group differences in model-based reasoning might instead be explained by group differences in the DSST. To correct for the DSST, we again calculated 1-tailed independentsamples $t$ tests after regressing out individual DSST scores. Although patients continued to be less model based, this correction removed the significant group difference $\left(t_{47}=1.13, p=0.13\right.$; fig. 5a: group differences in model-based scores, fig. 5b: model-based scores corrected for DSST).

\section{Discussion}

The current study suggests a disruption of model-based choice behavior in alcohol-dependent patients. Importantly, we found no difference in the strength of model-free choice tendencies, and the disruption appears to be present after nonreward outcomes only. Despite carefully matching patients for educational level, patients and controls differed on one measure of cognitive speed. Patients were less model based than controls even after controlling for this, but the difference was no longer significant $(\mathrm{p}=0.13$ ).

The results are in line with theories suggesting a shift from controlled, goal-directed (outcome-guided) to automatic, habitual (stimulus-guided) decision-making in substance dependence [8-11]. They also speak to the critical question of whether chronic drug intake affects the 
goal-directed or the habitual system, or both [13, 43]. Crucially, inflexible, habitual action tendencies in substance dependence could derive from either an overactive habit system or an underactive goal-directed system or a change in the balance between these two systems [44]. Nonsequential tasks, such as devaluation paradigms, cannot address this question as they do not allow specifications on whether habitual action tendencies rather rely on amplified stimulus-response associations (habit system) or on an impairment to update or modify action-outcome contingencies (goal-directed system), or a combination of both. Thus, we here used a Markov decision task which allows precise specifications on the individual contribution of each system on decision-making. The results presented here suggest mainly an effect on goal-directed choices, particularly after nonrewards. This is in line with data showing orbitofrontal $[45,46]$ and action-outcome impairments in substance dependence [13] and with accounts emphasizing the importance of reasserting goaldirected control after losses or punishment [7, 47-49]. Interestingly, administration of L-dopa in this task selectively enhances goal-directed action selection on unrewarded trials [50]. This may speak to the profound alterations in the dopaminergic system in addiction [5153]. Learning from nonrewards is known to rely on $D_{2}$ receptors in the indirect, inhibitory loop of the basal ganglia $[54,55]$. While this has mainly been examined in the context of habitual, prediction-error-based learning, Frank's model also provides routes for influences on the prefrontal cortex via internal actions that update working memory [56].

The study identified a difference in the DSST between patients matched for educational level. This finding is in line with other studies demonstrating deficits in the DSST in alcohol-dependent patients $[57,58]$ and addiction disorders are well known not only to relate to striatal changes, but also decreased activation in the prefrontal cortex [53]. This may hint at an effect of alcohol on more general measures of cognitive functioning [58]. Goal-directed control has also been associated with prefrontal areas $[27,59,60]$. This raises the question of whether drugs directly affect goal-directed choices or do so only secondarily to their effects on other, more general cognitive functions. It will be important to also correct carefully for other measures of global function, such as working memory [25]. Indeed, confounding factors from even further afield may play a role, too. Stress, for instance, is known to affect the deployment of the goal-directed system [61] by decreasing activity of prefrontal regions [62], and it is not entirely implausible that patients may have felt more

Decision-Making after Chronic Alcohol Intake stressed during the experiment than controls. Moreover, it has been demonstrated that trait impulsivity is linked to decreased goal-directed control [63]. Given the finding that self-reported impulsivity tends to be increased in substance-dependent subjects $[64,65]$, this might be another potential mediator for the effects reported here.

We did not find a difference in model-free learning. Very detailed computational theories map phasic dopamine signals decisively onto model-free learning [66-69]. Given the impact of drugs of abuse on dopamine [70], the absence of this finding is rather striking. However, the failure to observe an effect on model-free learning is a negative effect. Similarly, the study was powered to detect a group difference in either of the two components, but not a difference between them, and we therefore did not ask whether the difference in the model-based component was larger than the difference in the model-free component. Hence these findings need to be treated with caution and require replication. Furthermore, while animal studies have convincingly shown a shift towards model-free choices in addiction, and indeed with alcohol $[4,5,7]$, there have been only limited investigations to substantiate this in humans so far $[23,27]$. One reason may be that it has been difficult to measure these two systems in humans and often has required rather laborious tasks involving extended training $[71,72]$. While the current task is more subtle and measures devaluation in a more continuous way, one caveat is that it is not clear whether it fully differentiates between goal-directed and habitual components at a neurobiological level [31]. Moreover, it is yet unclear how different paradigms that have been designed to investigate dual-control mechanisms in humans indeed do examine a common psychological and neurobiological construct. Thus, further research should investigate within-subject correlations between performance in different dual-control tasks, such as paradigms that devalued outcomes by satiation $[24,61$, 71] or by omission [72] and sequential learning tasks such as the 2-step task [31] or even more complex Marcov decision tasks [73].

We found that patients earned slightly less than controls. As a goal-directed strategy earns more, we initially thought this would be explained by the measure of goaldirectedness. This was not so, suggesting that there might be further factors, possibly beyond the current modelfree/model-based account affecting subjects' performance on the task. However, this need not necessarily be the case and in the future could be addressed by fitting the computational models of Daw et al. [31] and examining specifically the softmax terms.

Neuropsychobiology 2014;70:122-13 
Finally, it is yet unclear whether disruption in modelbased control in alcohol-dependent subjects is caused by excessive chronic alcohol intake or rather reflects a predisposition to alcohol abuse. Evidence for alcohol-induced disruption of goal-directed behavior comes from demonstrations of devaluation insensitivity after chronic alcohol intake in rodents [4] and humans revealing disruption of goal-directed behavior [24] and inhibitory top-down control [74] after acute alcohol administration. As it has been shown that impairments in cognitive control serve as a vulnerability marker for an increased risk for substance dependence [65, 75-77], further research should use longitudinal designs in order to answer this chicken-and-egg question.
In conclusion, we have shown rather selective and specific effects of chronic alcohol intake on model-based reasoning, and highlighted that this might arise from the impact of chronic alcohol intake on more general cognitive functions. Further research should pay detailed attention to how impairments in the goal-directed system are related to prefrontal and cognitive functioning.

\section{Acknowledgment}

This work was supported by the German Research Foundation (Deutsche Forschungsgemeinschaft, FOR 1617: grants HE2597/141 and ZI1119/3-1 and DFG RA1047/2-1).

\section{References}

1 Balleine BW, Dickinson A: Goal-directed instrumental action: contingency and incentive learning and their cortical substrates. Neuropharmacology 1998;37:407-419.

-2 Sutton RS, Barto AG: Reinforcement Learning: An Introduction. Cambridge, MIT Press, 1998, p 1.

$\checkmark 3$ Daw ND, Niv Y, Dayan P: Uncertainty-based competition between prefrontal and dorsolateral striatal systems for behavioral control. Nat Neurosci 2005;8:1704-1711.

$\checkmark 4$ Dickinson A, Wood N, Smith JW: Alcohol seeking by rats: action or habit? Q J Exp Psychol B Comp Physiol Psychol 2002;55:331-348.

$\checkmark 5$ Hopf FW, Chang S-J, Sparta DR, Bowers MS, Bonci A: Motivation for alcohol becomes resistant to quinine adulteration after 3 to 4 months of intermittent alcohol self-administration. Alcohol Clin Exp Res 2010;34:1565-1573.

$\checkmark 6$ Wolffgramm J, Heyne A: From controlled drug intake to loss of control: the irreversible development of drug addiction in the rat. Behav Brain Res 1995;70:77-94.

7 Ostlund SB, Maidment NT, Balleine BW: Alcohol-paired contextual cues produce an immediate and selective loss of goal-directed action in rats. Frontiers in Integrative Neuroscience 2010;4:19.

8 Robbins TW, Everitt BJ: Drug addiction: bad habits add up. Nature 1999;398:567-570.

$>9$ Jentsch JD, Taylor JR: Impulsivity resulting from frontostriatal dysfunction in drug abuse: implications for the control of behavior by reward-related stimuli. Psychopharmacology 1999;146:373-390.

$>10$ Bechara A: Decision making, impulse control and loss of willpower to resist drugs: a neurocognitive perspective. Nat Neurosci 2005;8: 1458-1463.

-11 Belin D, Jonkman S, Dickinson A, Robbins TW, Everitt BJ: Parallel and interactive learning processes within the basal ganglia: relevance for the understanding of addiction. Behav Brain Res 2009;199:89-102.
12 Jentsch JD, Olausson P, De La Garza R 2nd, Taylor JR: Impairments of reversal learning and response perseveration after repeated, intermittent cocaine administrations to monkeys. Neuropsychopharmacology 2002;26: 183-190.

13 Schoenbaum G, Setlow B: Cocaine makes actions insensitive to outcomes but not extinction: implications for altered orbitofrontalamygdalar function. Cereb Cortex 2005;15: 1162-1169.

14 Park SQ, Kahnt T, Beck A, Cohen MX, Dolan RJ, Wrase J, Heinz A: Prefrontal cortex fails to learn from reward prediction errors in alcohol dependence. J Neurosci 2010;30:7749-7753.

15 Ersche KD, Roiser JP, Robbins TW, Sahakian BJ: Chronic cocaine but not chronic amphetamine use is associated with perseverative responding in humans. Psychopharmacology 2008;197:421-431.

16 Fillmore MT, Rush CR: Polydrug abusers display impaired discrimination-reversal learning in a model of behavioural control. J Psychopharmacol 2006;20:24-32.

17 Ersche KD, Roiser JP, Abbott S, Craig KJ, Muller U, Suckling J, Ooi C, Shabbir SS, Clark L, Sahakian BJ, Fineberg NA, Merlo-Pich EV, Robbins TW, Bullmore ET: Response perseveration in stimulant dependence is associated with striatal dysfunction and can be ameliorated by a $\mathrm{D}_{2 / 3}$ receptor agonist. Biol Psychiatry 2011;70:754-762.

-18 Lawrence AJ, Luty J, Bogdan NA, Sahakian BJ, Clark L: Impulsivity and response inhibition in alcohol dependence and problem gambling. Psychopharmacology 2009;207: $163-172$.

$>19$ Morein-Zamir S, Simon Jones P, Bullmore ET, Robbins TW, Ersche KD: Prefrontal hypoactivity associated with impaired inhibition in stimulant-dependent individuals but evidence for hyperactivation in their unaffected siblings. Neuropsychopharmacology 2013; 38:1945-1953.
20 Marhe R, Waters AJ, van de Wetering BJ, Franken IH: Implicit and explicit drug-related cognitions during detoxification treatment are associated with drug relapse: an ecological momentary assessment study. J Consult Clin Psychol 2013;81:1-12.

21 Wiers RW, Eberl C, Rinck M, Becker ES, Lindenmeyer J: Retraining automatic action tendencies changes alcoholic patients' approach bias for alcohol and improves treatment outcome. Psychol Sci 2011;22:490-497.

22 Hogarth L, Chase HW: Parallel goal-directed and habitual control of human drug-seeking: implications for dependence vulnerability. J Exp Psychol Anim Behav Processes 2011; 37:261-276.

23 Hogarth L, Field M, Rose AK: Phasic transition from goal-directed to habitual control over drug-seeking produced by conflicting reinforcer expectancy. Addict Biol 2013;18:8897.

24 Hogarth L, Attwood AS, Bate HA, Munafo MR: Acute alcohol impairs human goal-directed action. Biol Psychol 2012;90:154-160.

25 Otto AR, Gershman SJ, Markman AB, Daw ND: The curse of planning: dissecting multiple reinforcement-learning systems by taxing the central executive. Psychol Sci 2013;24: 751-761.

26 Guillot CR, Fanning JR, Bullock JS, McCloskey MS, Berman ME: Effects of alcohol on tests of executive functioning in men and women: a dose response examination. Exp Clin Psychopharmacol 2010;18:409-417.

27 Sjoerds Z, de Wit S, van den Brink W, Robbins TW, Beekman AT, Penninx BW, Veltman DJ: Behavioral and neuroimaging evidence for overreliance on habit learning in alcohol-dependent patients. Translat Psychiatry 2013; 3:e337.

28 Tricomi E, Balleine BW, O’Doherty JP: A specific role for posterior dorsolateral striatum in human habit learning. Eur J Neurosci 2009; 29:2225-2232. 
29 Bornstein AM, Daw ND: Multiplicity of control in the basal ganglia: computational roles of striatal subregions. Curr Opin Neurobiol 2011; 21:374-380.

-30 Rangel A, Hare T: Neural computations associated with goal-directed choice. Curr Opin Neurobiol 2010;20:262-270.

-31 Daw ND, Gershman SJ, Seymour B, Dayan P, Dolan RJ: Model-based influences on humans' choices and striatal prediction errors. Neuron 2011;69:1204-1215.

-32 Corbit LH, Nie H, Janak PH: Habitual alcohol seeking: time course and the contribution of subregions of the dorsal striatum. Biol Psychiatry 2012;72:389-395.

33 Otto AR, Raio CM, Chiang A, Phelps EA, Daw ND: Working-memory capacity protects model-based learning from stress. Proc Natl Acad Sci USA 2013;110:20941-20946.

-34 Smittenaar P, FitzGerald TH, Romei V, Wright ND, Dolan RJ: Disruption of dorsolateral prefrontal cortex decreases model-based in favor of model-free control in humans. Neuron 2013;80:914-919.

35 First M, Spitzer R, Gibbon M, Williams J: Structured clinical interview for DSM-IV-TR axis I disorders, research version, patient edition with psychotic screen (SCID-I/P W/PSY SCREEN). New York, New York State Psychiatric Institute, Biometrics Research, 2001.

- 36 Brainard DH: The psychophysics toolbox. Spatial Vision 1997;10:433-436.

37 Pelli DG: The videotoolbox software for visual psychophysics: transforming numbers into movies. Spatial Vision 1997;10:437-442.

38 Matlab: Version 7.4.0 (r2007a). Natick, The MathWorks Inc, 2007.

39 Schmidt K-HMP: Wortschatztest (WST). Weinheim, Beltz, 1992.

40 Wechsler D: WAIS-R manual. New York, Psychol Corp, 1981.

41 Army individual test battery. Manual of directions and scoring. Washington, War Department, Adjutant's Generals Office, 1944.

-42 Morris JC, Heyman A, Mohs RC, Hughes JP, van Belle G, Fillenbaum G, Mellits ED, Clark C: The Consortium to Establish a Registry for Alzheimer's Disease (CERAD). I. Clinical and neuropsychological assessment of Alzheimer's disease. Neurology 1989;39:1159-1165.

-43 Simon D, Daw N: Dual-system learning models and drugs of abuse; in Gutkin B, Ahmed SH (eds): Computational Neuroscience of Drug Addiction. New York, Springer, 2012, vol 10, pp 145-161.

-44 Redish AD, Jensen S, Johnson A: A unified framework for addiction: vulnerabilities in the decision process. Behav Brain Sci 2008;31:415437.

-45 Lucantonio F, Stalnaker TA, Shaham Y, Niv Y, Schoenbaum G: The impact of orbitofrontal dysfunction on cocaine addiction. Nat Neurosci 2012;15:358-366.

-46 Lucantonio F, Caprioli D, Schoenbaum G: Transition from 'model-based' to 'model-free' behavioral control in addiction: involvement of the orbitofrontal cortex and dorsolateral striatum. Neuropharmacology 2014;76:407415.
47 Chen BT, Yau HJ, Hatch C, Kusumoto-Yoshida I, Cho SL, Hopf FW, Bonci A: Rescuing cocaine-induced prefrontal cortex hypoactivity prevents compulsive cocaine seeking. Nature 2013;496:359-362.

48 Vanderschuren LJ, Everitt BJ: Drug seeking becomes compulsive after prolonged cocaine selfadministration. Science 2004;305:1017-1019.

49 Deroche-Gamonet V, Belin D, Piazza PV: Evidence for addiction-like behavior in the rat. Science 2004;305:1014-1017.

50 Wunderlich K, Smittenaar P, Dolan RJ: Dopamine enhances model-based over model-free choice behavior. Neuron 2012;75:418-424.

51 Heinz A, Dufeu P, Kuhn S, Dettling M, Gräf K, Kürten I, Rommelspacher H, Schmidt LG: Psychopathological and behavioral correlates of dopaminergic sensitivity in alcohol-dependent patients. Arch Gen Psychiatry 1996;53:11231128.

52 Heinz A, Siessmeier T, Wrase J, Buchholz HG, Gründer G, Kumakura Y, Cumming $P$, Schreckenberger M, Smolka MN, Rösch F, Mann K, Bartenstein P: Correlation of alcohol craving with striatal dopamine synthesis capacity and $D_{2 / 3}$ receptor availability: a combined $\left[{ }^{18} \mathrm{~F}\right] \mathrm{DOPA}$ and $\left[{ }^{18} \mathrm{~F}\right] \mathrm{DMFP}$ pet study in detoxified alcoholic patients. Am J Psychiatry 2005; 162:1515-1520.

53 Volkow ND, Fowler JS, Wang GJ, Baler R, Telang F: Imaging dopamine's role in drug abuse and addiction. Neuropharmacology 2009;56(suppl 1):3-8.

54 Frank MJ, Seeberger LC, O’Reilly RC: By carrot or by stick: cognitive reinforcement learning in parkinsonism. Science 2004;306:1940-1943.

55 Maia TV, Frank MJ: From reinforcement learning models to psychiatric and neurological disorders. Nat Neurosc 2011;14:154-162.

56 Frank MJ: Dynamic dopamine modulation in the basal ganglia: A neurocomputational account of cognitive deficits in medicated and nonmedicated parkinsonism. J Cogn Neurosci 2005; 17:51-72.

57 Davies SJ, Pandit SA, Feeney A, Stevenson BJ, Kerwin RW, Nutt DJ, Marshall EJ, Boddington $\mathrm{S}$, Lingford-Hughes A: Is there cognitive impairment in clinically 'healthy' abstinent alcohol dependence? Alcohol Alcohol 2005;40: 498-503.

58 Moselhy HF, Georgiou G, Kahn A: Frontal lobe changes in alcoholism: a review of the literature. Alcohol Alcohol 2001;36:357-368.

59 Killcross S, Coutureau E: Coordination of actions and habits in the medial prefrontal cortex of rats. Cereb Cortex 2003;13:400-408.

60 Duncan J, Emslie H, Williams P, Johnson R, Freer C: Intelligence and the frontal lobe: the organization of goal-directed behavior. Cogn Psychol 1996;30:257-303.

61 Schwabe L, Wolf OT: Stress prompts habit behavior in humans. J Neuroscience 2009;29: 7191-7198.
62 Schwabe L, Tegenthoff M, Hoffken O, Wolf OT: Simultaneous glucocorticoid and noradrenergic activity disrupts the neural basis of goal-directed action in the human brain. J Neurosci 2012;32:10146-10155.

- 63 Hogarth L, Chase HW, Baess K: Impaired goaldirected behavioural control in human impulsivity. Q J Exp Psychol 2012;65:305-316.

64 Beck A, Schlagenhauf F, Wüstenberg T, Hein J, Kienast T, Kahnt T, Schmack K, Hägele C, Knutson B, Heinz A, Wrase J: Ventral striatal activation during reward anticipation correlates with impulsivity in alcoholics. Biol Psychiatry 2009;66:734-742.

65 De Wit H: Impulsivity as a determinant and consequence of drug use: a review of underlying processes. Addict Biol 2009;14:22-31.

66 Montague PR, Dayan P, Sejnowski TJ: A framework for mesencephalic dopamine systems based on predictive hebbian learning. J Neurosci 1996;16:1936-1947.

67 Schultz W: Dopamine neurons and their role in reward mechanisms. Curr Opin Neurobiol 1997;7:191-197.

68 Steinberg EE, Keiflin R, Boivin JR, Witten IB, Deisseroth K, Janak PH: A causal link between prediction errors, dopamine neurons and learning. Nat Neurosci 2013;16:966-973.

69 Bayer HM, Glimcher PW: Midbrain dopamine neurons encode a quantitative reward prediction error signal. Neuron 2005;47:129-141.

70 Dayan P: Dopamine, reinforcement learning, and addiction. Pharmacopsychiatry 2009; 42(suppl 1):S56-S65.

71 Valentin VV, Dickinson A, O’Doherty JP: Determining the neural substrates of goal-directed learning in the human brain. J Neurosci 2007;27:4019-4026.

72 De Wit S, Corlett PR, Aitken MR, Dickinson A, Fletcher PC: Differential engagement of the ventromedial prefrontal cortex by goal-directed and habitual behavior toward food pictures in humans. J Neurosci 2009;29:11330-11338.

73 Lee SW, Shimojo S, O’Doherty JP: Neural computations underlying arbitration between model-based and model-free learning. Neuron 2014;81:687-699.

74 Field M, Wiers RW, Christiansen P, Fillmore MT, Verster JC: Acute alcohol effects on inhibitory control and implicit cognition: implications for loss of control over drinking. Alcohol Clin Exp Res 2010;34:1346-1352.

75 Ersche KD, Jones PS, Williams GB, Turton AJ, Robbins TW, Bullmore ET: Abnormal brain structure implicated in stimulant drug addiction. Science 2012;335:601-604.

76 Dalley JW, Fryer TD, Brichard L, Robinson ES, Theobald DE, Laane K, Pena Y, Murphy ER, Shah Y, Probst K, Abakumova I, Aigbirhio FI, Richards HK, Hong Y, Baron JC, Everitt BJ, Robbins TW: Nucleus accumbens $\mathrm{D}_{2 / 3}$ receptors predict trait impulsivity and cocaine reinforcement. Science 2007;315:1267-1270.

-77 Flagel SB, Robinson TE, Clark JJ, Clinton SM, Watson SJ, Seeman P, Phillips PE, Akil H: An animal model of genetic vulnerability to behavioral disinhibition and responsiveness to reward-related cues: implications for addiction. Neuropsychopharmacology 2010;35:388-400. 


\section{Erratum}

In the article by Sebold et al., entitled 'Model-based and model-free decisions in alcohol dependence' [Neuropsychobiology 2014;70:122-131, DOI: 10.1159/000362840], the names of the following two authors should correctly read:

Stephan Nebe and Michael N. Smolka

The following affiliations should correctly read:

${ }^{b}$ Department of Psychology, Humboldt-Universität zu Berlin, Berlin, Germany

${ }^{\mathrm{d}}$ Department of Psychiatry and Neuroimaging Center, Technische Universität Dresden, Dresden, Germany

${ }^{\mathrm{e}}$ Department of Psychiatry and Psychotherapy, University Hospital Carl Gustav Carus, Technische Universität Dresden, Dresden, Germany

${ }^{g}$ Translational Neuromodeling Unit, Institute for Biomedical Engineering, University of Zurich and Swiss Federal Institute of Technology (ETH) Zurich, Zurich, Switzerland 